\title{
High performances stack microstrip antenna for under water communications at 2.4 $\mathbf{G H z}$
}

\begin{abstract}
This paper shows a new compact size stack microstrip antenna which is designed and simulated to improve and meet the radiation characteristics requirements in normal water. Thus, a rectangular patch with three periodic slots as Photonic Band Gap (PBG) have been cut from the patch. Furthermore, two elliptical rings at the back is resonating at $2.4 \mathrm{GHz}$ for IEEE $802.11 \mathrm{~b} / \mathrm{g} / \mathrm{h}$ standards and WLAN communications, with miniaturized dimensions of $45 \times 40 \mathrm{~mm}$. Return loss, VSWR, radiation efficiency and gain have been exploited in order to define antennas' performance in both air and normal water.
\end{abstract}

Keyword: Efficiency; Gain; PBG; Shrunk size; Stack 